Pacific Journal of Mathematics

BOUNDS FOR EIGENVALUES AND GENERALIZED
CONVEXITY 


\section{BOUNDS FOR EIGENVALUES AND GENERALIZED CONVEXITY}

\section{Dallas O. BANKS}

1. Introduction. The eigenvalue problem associated with a vibrating string which is under unit tension has a nonnegative integrable density function $\rho$ defined for $x \in[a, b]$ and which is held elastically at its ends is

$$
\begin{gathered}
u^{\prime \prime}+\lambda \rho(x) u=0, \\
u^{\prime}(a)-h_{a} u(a)=u^{\prime}(b)+h_{b} u(b)=0
\end{gathered}
$$

where $0 \leqq h_{a}, h_{b} \leqq \infty$. The limiting case $h_{a} \rightarrow \infty, h_{b} \rightarrow \infty$ corresponds to the fixed end point problem. In general, the eigenvalues of the system (1.1) are nonnegative, simple and depend on the function $\rho$. We denote them accordingly by

$$
0<\lambda_{1}[\rho]<\lambda_{2}[\rho]<\cdots<\lambda_{n}[\rho]<\cdots .
$$

We consider the problem of finding uniform upper and lower bounds for $\lambda_{n}[\rho] \quad(n=1,2, \cdots)$ when $\rho$ is restricted to belong to a specific set of functions. In particular, we consider sets of functions $\rho$ which are either convex or concave in the following generalized sense.

Let

$$
L(y) \equiv\left(r(x) y^{\prime}\right)^{\prime}-p(x) y, \quad x \in[a, b]
$$

where $r$ and $p$ are real-valued continuous functions on $[a, b]$ with $r(x)>0$ and $r \in C^{\prime}$ on $[a, b]$. Furthermore, we consider only those equations of the form (1.2) whose solutions satisfy the

Existence Property. There exists a unique solution $y$ of (1.2) through the points $\left(x_{1}, y_{1}\right),\left(x_{2}, y_{2}\right)$ where $a \leqq x_{1}<x_{2} \leqq b$ and $y_{1}, y_{2}$ are arbitary real numbers. We denote the values of $y$ by $y(x)=$ $y\left(x ; x_{1}, y_{1} ; x_{2}, y_{2}\right)$.

Definition. A real function $\rho$ is $\operatorname{sub}-(L)$ on $[a, b]$ if for arbitrary $x_{1}, x_{2}$ such that $a \leqq x_{1}<x_{2} \leqq b$, we have

$$
\rho(x) \leqq y\left(x ; x_{1}, \rho\left(x_{1}\right) ; x_{2}, \rho\left(x_{2}\right)\right), \quad x \in\left[x_{1}, x_{2}\right] .
$$

$\rho$ is $\operatorname{super}-(L)$ if

$$
\rho(x) \geqq y\left(x ; x_{1}, \rho\left(x_{1}\right) ; x_{2}, \rho\left(x_{2}\right)\right), \quad x \in\left[x_{1}, x_{2}\right] .
$$

Received December 6, 1962. 
In the case $r(x) \equiv 1, p(x) \equiv 0, x \in(a, b)$, this reduces to ordinary convexity and concavity. Sub- $(L)$ and super- $(L)$ functions have been studied by Bonsall [6], Peixoto [11] and Reid [12]. Sub- $(L)$ functions are a special case of the sub- $(F)$ functions investigated by Beckenbach [3].

In the next section, we give some integral representations of sub$(L)$ and super- $(L)$ functions. These are used in $\S 3$ to find $\inf _{\rho \in E_{i}} \lambda_{1}[\rho]$ $(i=1,2)$ where

$$
E_{1}=\left\{\rho \mid \int_{a}^{b} \rho(x) d x=M, \rho \operatorname{sub}-(L) \text { on }[a, b]\right\}
$$

and

$$
E_{2}=\left\{\rho \mid \int_{a}^{b} \rho(x) d x=M, \quad \rho \text { super- }(L) \text { on }[a, b]\right\}
$$

and to find the functions for which these bounds are attained. In $\S 4$, we discuss the problem of finding $\inf _{E_{i}} \lambda_{n}[\rho]$ for each of the sets $E_{i}(i=1,2)$ for any $n$. In the last two sections we consider the $\sup _{B_{i}} \lambda_{n}[\rho](i=1,2)$ and the functions $\rho$ for which these bounds are attained. Also some generalizations to other eigenvalue problems are discussed.

The results presented in this paper are a generalization of some of those described in [1] and [2] where the sup $\lambda_{n}[\rho]$ and inf $\lambda_{n}[\rho]$ are studied for density functions which are convex or concave. For if we let $r(x) \equiv 1$ and $p(x) \equiv 0(x \in[a, b])$ in (1.2), we get $L(y)=y^{\prime \prime}$ so that in this case a sub- $(L)$ function is a convex function and a super- $(L)$ function is a concave function. For other results of the same nature as those presented here see [5], [10] and [13].

2. Integral representations. We now show that sub- $(L)$ and super$(L)$ functions may be represented as integrals. This is most easily done if we use the following lemma due to W. T. Reid [12].

LEMMA (Reid). There exists a solution $y_{0}$ of $L(y)=0$ on $[a, b]$ such that $y_{0}(x)>0$; moreover, if $x_{0} \in[a, b]$ and $x(t)$ is defined by

$$
t(x)=\int_{x_{0}}^{x} \eta_{0}(z) d z, \eta_{0}(z)=1 / r(z) y_{0}^{2}(z), \quad(\alpha<t<\beta)
$$

where $\alpha=t(a), \beta=t(b)$, then $\rho$ is sub-(L) (super- $(L)$ ) on $[a, b]$ if and only if $\rho^{*}(t)=\rho(x(t)) / y_{0}(x(t))$ is convex (concave) on $[\alpha, \beta]$.

We denote the derivative from the right of the function $\rho$ by $\rho_{+}^{\prime}$ and the left derivative by $\rho_{-}^{\prime}$.

THEOREM 2.1. Let $\rho$ be any nonnegative sub- $(L)$ function on $[a, b]$. 
(i) If $\rho(a)=0$,

$$
\rho(x)=y_{0}(x) \int_{a}^{b} g_{1}(x, \xi) d f_{1}(\xi)
$$

where

$$
g_{1}(x, \xi)= \begin{cases}0, & a \leqq x \leqq \xi \leqq b \\ \int_{\xi}^{x} \eta_{0}(z) d z, & a \leqq \xi \leqq x \leqq b\end{cases}
$$

and

$$
f_{1}(\xi)=\left\{\begin{array}{lr}
r(\xi) y_{0}^{2}(\xi)\left(\rho(\xi) / y_{0}(\xi)\right)_{-}^{\prime}, & a<\xi \leqq b, \\
0, & \xi=a .
\end{array}\right.
$$

Moreover, $f_{1}$ is an increasing function.

(ii) If $\rho(b)=0$,

$$
\rho(x)=y_{0}(x) \int_{a}^{b} g_{2}(x, \xi) d f_{2}(\xi)
$$

where

$$
g_{2}(x, \xi)= \begin{cases}\int_{x}^{\xi} \eta_{0}(z) d z, & a \leqq x \leqq \xi \leqq b, \\ 0, & a \leqq \xi \leqq x \leqq b,\end{cases}
$$

and

$$
f_{2}(\xi)=\left\{\begin{array}{lr}
r(\xi) y_{0}^{2}(\xi)\left(\rho(\xi) / y_{0}(\xi)\right)_{+}^{\prime}, & a \leqq \xi<b, \\
0, & \xi=b .
\end{array}\right.
$$

Moreover, $f_{2}(\xi)$ is increasing.

(iii) Any nonnegative sub- $(L)$ function on $[a, b]$ may be written in the form $\rho=\rho_{1}+\rho_{2}$ where $\rho_{1}(a)=0$ and $\rho_{2}(b)=0$ with both $\rho_{1}$ and $\rho_{2}$ nonnegative and sub- $(L)$ on $[a, b]$.

Proof. To prove (i), we integrate by parts and use (2.1) to get

$$
\int_{a}^{b} g_{1}(x, \xi) d f_{1}(\xi)=\left.f_{1}(\xi) \int_{\xi}^{x} \eta_{0}(z) d z\right|_{a} ^{\xi}+\int_{a}^{x}\left(\rho(\xi) / y_{0}(\xi)\right)_{-}^{\prime} d \xi .
$$

The first term on the right vanishes. We assert that the integral on the right is equal to $\rho(x) / y_{0}(x)$. For the change of variables (2.1) yields the integral

$$
\int_{\infty}^{t}\left[\rho(x(\tau)) / y_{0}(x(\tau))\right]_{-}^{\prime} d \tau
$$

By Reid's lemma, the integrand of this integral is a bounded convex 
function in $[\alpha, t]$ for all $\alpha \leqq t \leqq \beta$. Hence, it is absolutely continuous and the assertion follows. To show that $f_{1}$ is an increasing function, we note that by Reid's lemma $\rho^{*}(t)=\rho(x(t)) / y_{0}(x(t))$ is convex on $[\alpha, \beta]$ and therefore $\left[\rho^{*}(t)\right]_{-}^{\prime}$ is increasing there. From (2.1) we have

$$
\left[\rho^{*}(t)\right]_{-}^{\prime}=\left[\rho(x) / y_{0}(x)\right]_{-}^{\prime} x^{\prime}(t)=f_{1}(x) .
$$

Since $x$ is an increasing function of $t$ it follows that $f_{1}(x)$ is increasing.

The proof of (ii) follows similarly. To prove (iii) we consider any nonnegative sub- $(L)$ function $\rho$. By Reid's lemma $\rho^{*}(t)=\rho(x(t)) / y_{0}(x(t))$ is convex and nonnegative. Then there is a minimum point $\tau \in[\alpha, \beta]$ of $\rho^{*}$. It is easily verified that $\rho^{*}(t)=\rho_{1}^{*}(t)+\rho_{2}^{*}(t)$ where

$$
\rho_{1}^{*}(t)= \begin{cases}\rho^{*}(\tau)(t-\alpha) /(\beta-\alpha), & \alpha \leqq t \leqq \tau, \\ \rho^{*}(t)-\rho^{*}(\tau)(\beta-t) /(\beta-\alpha), & \tau \leqq t \leqq \beta,\end{cases}
$$

and

$$
\rho_{2}^{*}(t)= \begin{cases}\rho^{*}(t)-\rho^{*}(\tau)(t-\alpha) /(\beta-\alpha), & \alpha \leqq t \leqq \tau, \\ \rho^{*}(\tau)(\beta-t) /(\beta-\alpha), & \tau \leqq t \leqq \beta .\end{cases}
$$

Clearly $\rho_{1}^{*}$ and $\rho_{2}^{*}$ are nonnegative convex functions with $\rho_{1}^{*}(\alpha)=0$ and $\rho_{2}^{*}(\beta)=0$. Again by Reid's lemma, we may transform $\rho_{1}^{*}$ and $\rho_{2}^{*}$. If we let $\tau=\int_{a}^{\xi} \eta_{0}(z) d z, \rho_{1}^{*}(t)$ becomes

$$
\rho_{1}(x)= \begin{cases}\rho(\xi) y_{0}(x) \int_{a}^{x} \eta_{0}(z) d z / y_{0}(\xi) \int_{a}^{b} \eta_{0}(z) d z, & a \leqq x \leqq \xi, \\ \rho(x)-\rho(\xi) y_{0}(x) \int_{x}^{b} \eta_{0}(z) d z / y_{0}(\xi) \int_{a}^{b} \eta_{0}(z) d z, & \xi \leqq x \leqq b,\end{cases}
$$

and

$$
\rho_{2}(x)= \begin{cases}\rho(x)-\rho(\xi) y_{0}(x) \int_{a}^{x} \eta_{0}(z) d z / y_{0}(\xi) \int_{a}^{b} \eta_{0}(z) d z, & a \leqq x \leqq \xi, \\ \rho(\xi) y_{0}(x) \int_{x}^{b} \eta_{0}(z) d z / y_{0}(\xi) \int_{a}^{b} \eta_{0}(z) d z, & \xi \leqq x \leqq b,\end{cases}
$$

where $\rho_{1}$ and $\rho_{2}$ are nonnegative sub- $(L)$ functions on $[a, b]$.

For nonnegative super- $(L)$ functions, we have

THEOREM 2.2 Any nonnegative super-(L) function $\rho$ defined on. $[a, b]$ has the integral representation

$$
\begin{aligned}
\rho(x)= & y_{0}(x)\left\{\rho(a) \int_{x}^{b} \eta_{0}(z) d z / y_{0}(a) \int_{a}^{b} \eta_{0}(z) d z\right. \\
& \left.+\rho(b) \int_{a}^{x} \eta_{0}(z) d z / y_{0}(b) \int_{a}^{b} \eta_{0}(z) d z\right\}+y_{0}(x) \int_{a}^{b} g(x, \xi) \dot{d} f(\xi)
\end{aligned}
$$




$$
g(x, \xi)= \begin{cases}\int_{a}^{x} \eta_{0}(z) d z \int_{\xi}^{b} \eta_{0}(z) d z / \int_{a}^{b} \eta_{0}(z) d z, & a \leqq x \leqq \xi \leqq b, \\ \int_{x}^{b} \eta_{0}(z) d z \int_{a}^{\xi} \eta_{0}(z) d z / \int_{a}^{b} \eta_{0}(z) d z, & a \leqq \xi \leqq x \leqq b,\end{cases}
$$

and

$$
f(\xi)=\left\{\begin{array}{lr}
-r(\xi) y_{0}^{2}(\xi)\left[\rho(\xi) / y_{0}(\xi)\right]_{-}^{\prime}, & a<\xi \leqq b, \\
\lim _{\xi \rightarrow a+} f(\xi), & \xi=a .
\end{array}\right.
$$

Moreover, $f(\xi)$ is increasing.

Proof. Integration by parts yields

$$
\begin{aligned}
\int_{a}^{b} g(x, \xi) d f(\xi)= & {\left[\int_{x}^{b} \eta_{0}(z) d z / \int_{a}^{b} \eta_{0}(z) d z\right] \int_{a}^{x}\left[\rho(\xi) / y_{0}(\xi)\right]_{-}^{\prime} d \xi } \\
& -\left[\int_{a}^{x} \eta_{0}(z) d z / \int_{a}^{b} \eta_{0}(z) d z\right] \int_{x}^{b}\left[\rho(\xi) / y_{0}(\xi)\right]_{-}^{\prime} d \xi .
\end{aligned}
$$

As in the proof of Theorem 2.1, $\rho(\xi) / y_{0}(\xi)$ is absolutely continuous and (2.7) follows. The monotonicity of $f(\xi)$ may be verified in the same manner as the monotonicity of $f_{1}(\xi)$ in Theorem 2.1.

3. Lower bounds for $\lambda_{1}[\rho]$. We look for $\inf _{\rho \in E_{1}} \lambda_{1}[\rho]$ where $E_{1}=\left\{\rho \mid \rho\right.$ is sub- $(L)$ on $\left.[a, b], \int_{a}^{b} \rho(x) d x=M\right\}$. Before stating our result, we define

$$
G_{1}(x, \xi)=y_{0}(x) g_{1}(x, \xi) / N_{1}(\xi), \quad x \in[a, b]
$$

where $g_{1}(x, \xi)$ is defined by $(2.4)$ and $N_{1}(\xi)=\int_{a}^{b} y_{0}(x) g_{1}(x, \xi) d x$. Since $y_{0}$ satisfies $L\left(y_{0}\right)=0$, it is easily verified that $L\left(G_{1}\right)=0$ for each fixed $\xi$ except at $x=\xi$. Furthermore, $\int_{a}^{b} G_{1}(x, \xi) d x=1$. Similarly we define

$$
G_{2}(x, \xi)=y_{0}(x) g_{2}(x, \xi) / N_{2}(\xi), \quad x \in[a, b]
$$

where $g_{2}(x, \xi)$ is defined by $(2.6)$ and $N_{2}(\xi)=\int_{a}^{b} y_{0}(x) g_{2}(x, \xi) d x$. Clearly $L\left(G_{2}\right)=0$ and $\int_{a}^{b} G_{2}(x, \xi) d x=1$ as in the case $G_{1}(x, \xi)$.

THEOREM 3.1. Let $\lambda_{1}[\rho]$ be the lowest eigenvalue of a string of length $(b-a)$ with elastically held ends and density $\rho(x), x \in[a, b]$. If $\rho$ is a sub- $(L)$ function on $[a, b]$ where $L(y)$ is defined by (1.2), then

$$
\lambda_{1}[\rho] \int_{a}^{b} \rho(x) d x \geqq \lambda_{0}
$$

where 


$$
\lambda_{0}=\min \left\{\inf _{\xi \in[a, b]} \lambda_{1}\left[G_{1}\right], \inf _{\xi \in[a, b]} \lambda_{1}\left[G_{2}\right]\right\}
$$

with $G_{1}$ defined by (3.1) and $G_{2}$ defined by (3.2). If $\rho$ is also an increasing function, then

$$
\lambda_{0}=\inf _{\xi} \lambda_{1}\left[G_{1}\right]
$$

The proof of this theorem depends on the following lemma.

LEMMA 3.1. If $\rho(x)$ of (1.1) can be expressed as

$$
\rho(x)=\int_{a}^{b} K(x, \xi) N(\xi) d f(\xi)
$$

where $f$ is increasing on $[a, b], N$ is a nonnegative continuous function on $[a, b]$ and $K$ is a nonnegative function with $\int_{a}^{b} K(x, \xi) d x=1$, then

$$
\lambda_{1}[\rho] \int_{a}^{b} \rho(x) d x \geqq \inf _{\xi \in[a b]} \lambda_{1}[K] .
$$

A proof of this is given in [1], page 441, for the fixed boundary conditions $u(a)=u(b)=0$. The proof for the more general boundary conditions of (1.1) is a trivial generalization of the result presented there.

Proof of Theorem 3.1. By Theorem 2.1, $\rho=\rho_{1}+\rho_{2}$ where $\rho_{1}$ and $\rho_{2}$ are nonnegative sub- $(L)$ functions with $\rho_{1}(a)=0$ and $\rho_{2}(b)=0$. Let $\mu_{1}=\int_{a}^{b} \rho_{1}(x) d x$ and $\mu_{2}=\int_{a}^{b} \rho_{2}(x) d x$ so that $\mu_{1}+\mu_{2}=\int_{a}^{b} \rho(x) d x=M$. We now use the minimal characterization of lowest eigenvalue of (1.1) to prove our theorem. It is well known [7], p. 127, and [8], p. 402, that $\lambda_{1}[\rho]=\min _{v} R[\rho, v]$ where the minimum may be taken over all piecewise smooth functions $v$ which satisfy the boundary conditions of (1.1) and $R[\rho, v]$ is the Rayleigh quotient

$$
R[\rho, v]=\frac{\int_{a}^{b} v^{\prime 2}(x) d x+h_{a} v^{2}(a)+h_{b} v^{2}(b)}{\int_{a}^{b} \rho(x) v^{2}(x) d x}
$$

If either $h_{a}=\infty$ or $h_{b}=\infty$, the corresponding terms in the numerator do not appear. We note that $J[\rho, v]=1 / R[\rho, v]$ is a linear functional in $\rho$ and in particular $J[\rho, v]=J\left[\rho_{1}, v\right]+J\left[\rho_{2}, v\right]$ so that

$$
\begin{aligned}
1 / \lambda_{1}[\rho] & =\max _{v} J[\rho, v] \\
& \leqq \max _{v} J\left[\rho_{1}, v\right]+\max _{v} J\left[\rho_{2}, v\right] \\
& =1 / \lambda_{1}\left[\rho_{1}\right]+1 / \lambda_{1}\left[\rho_{2}\right] .
\end{aligned}
$$


(For other inequalities of this nature, see [9].) Now $\rho_{1}$ is an increasing sub- $(L)$ function on $[a, b]$ so that Lemma 3.1 implies $\lambda_{1}\left[\rho_{1}\right] \mu_{1} \geqq \inf _{\xi} \lambda_{1}\left[G_{1}\right]$. Similarly, $\lambda_{1}\left[\rho_{2}\right] \mu_{2} \geqq \inf _{\xi} \lambda_{1}\left[G_{2}\right]$. Hence it follows that

$$
\begin{aligned}
1 / \lambda_{1}[\rho] & \leqq \mu_{1} / \inf _{\xi} \lambda_{1}\left[G_{1}\right]+\mu_{2} / \inf _{\xi} \lambda_{1}\left[G_{2}\right] \\
& \leqq\left(\mu_{1}+\mu_{2}\right) / \min \left\{\inf _{\xi} \lambda_{1}\left[G_{1}\right], \inf _{\xi} \lambda_{1}\left[G_{2}\right]\right\}
\end{aligned}
$$

and (3.3) is proved.

Finally, we note that if $\rho$ is increasing so that $\rho_{2}(x) \equiv 0, x \in[a, b]$, then $\mu_{2}=0$ and we have

$$
\lambda_{1}(\rho) \int_{a}^{b} \rho(x) d x \geqq \inf _{\xi} \lambda_{1}\left[G_{1}\right] .
$$

We will now apply this theorem to some special cases. We make use of the following

LEMMA 3.2. Let $g$ and $h$ be nonnegative integrable functions defined on $[a, b]$ and let $f$ be nonnegative, continuous and increasing on $[a, b]$. Let $c \in(a, b)$ be such that $g(x) \geqq h(x)$ for $x \in[a, c)$ and $g(x) \leqq h(x)$ for $x \in(c, b]$. Then

$$
\int_{a}^{b} g(x) d x=\int_{a}^{b} h(x) d x
$$

implies

$$
\int_{a}^{b} g(x) f(x) d x \leqq \int_{a}^{b} h(x) f(x) d x .
$$

If $f$ is decreasing, then the inequality is reversed.

This result is proved in [1] and in a more general form in [4].

We first apply our theorem to the case where the boundary conditions of $(1.1)$ are $u^{\prime}(a)-h_{a} u(a)=0, u^{\prime}(b)=0, h_{a}>0$. For these boundary conditions it is easily seen that the first eigenfunction $u_{1}$ of (1.1) is a strictly increasing function. By Lemma 3.2, for any positive monotone function $u$ on $(a, b)$ we have

$$
\int_{a}^{b} G_{2}(x, \xi) u^{2}(x) d x \leqq \int_{a}^{b} G_{1}(x, \xi) u^{2}(x) d x
$$

and

$$
\int_{a}^{b} G_{1}\left(x, \xi_{1}\right) u^{2}(x) d x \leqq \int_{a}^{b} G_{1}\left(x, \xi_{2}\right) u^{2}(x) d x
$$

for any pair of numbers $\xi_{1}, \xi_{2}$ such that $a \leqq \xi_{1}<\xi_{2} \leqq b$. It then follows from this and (3.5) that $R\left[G_{2}, u\right] \geqq R\left[G_{1}, u\right]$ and also that 
$R\left[G_{1}, u\right]$ is a decreasing function of $\xi$. Hence, we may conclude that

$$
\lambda_{1}[\rho] \int_{a}^{b} \rho(x) d x \geqq \lim _{\xi \rightarrow b} \lambda_{1}\left[G_{1}\right] .
$$

It may be verified that this limit is $h_{a} /\left[1+(b-a) h_{a}\right]$.

We now consider the case where $r$ and $p$ of $L(y)$ are symmetric with respect to $x=(a+b) / 2$. If the boundary conditions of (1.1) are of the special form $h_{a}=h_{b}$, then $\lambda_{1}\left[G_{1}\right]$ at a particular value of $\xi=\xi_{1}$ and $\lambda_{1}\left[G_{2}\right]$ at $\xi=b+a-\xi_{1}$ have the same value. Thus, $\inf \lambda_{1}\left[G_{2}\right]=\inf \lambda_{1}\left[G_{1}\right]$. For the particular case, $L(y)=y^{\prime \prime}$ and the boundary conditions $u(a)=u(b)=0$, we get

$$
\lambda_{1}[\rho] \int_{a}^{b} \rho(x) d x=\inf _{\xi} \lambda_{1}[q]
$$

where

$$
q(x, \xi)= \begin{cases}0, & a \leqq x \leqq \xi \leqq b \\ 2(x-\xi) /(b-\xi)^{2}, & a \leqq \xi \leqq x \leqq b\end{cases}
$$

This was proved directly in [1].

Finally, as a special case of Theorem 3.1, we have

THEOREM 3.2. Let $\lambda_{1}[\rho]$ be the lowest eigenvalue of a string of length $(b-a)$ with fixed end points. If the density function $\rho$ is sub- $(L)$ on $[a, b]$ where $L(y)=\left(r(x) y^{\prime}\right)^{\prime}$ with $r(x)>0, r^{\prime}(x) \geqq 0$ and $r^{\prime}$ continuous on $[a, b]$, then

$$
\lambda_{1}[\rho] \int_{a}^{b} \rho(x) d x \geqq \lambda_{0}
$$

where $\lambda_{0}=\min _{\xi} \lambda_{1}\left[G_{1}\right]$ and

$$
G_{1}(x, \xi)= \begin{cases}0, & a \leqq x \leqq \xi \leqq b, \\ \int_{\xi}^{x}[1 / r(z)] d z / \int_{\xi}^{b}[(b-z) / r(z)] d z, & a \leqq \xi \leqq x \leqq b .\end{cases}
$$

Proof. Applying Theorem 3.1 with $y_{0}(x) \equiv 1$, we get the lower bound (3.4) where $G_{1}$ has values (3.8) and $G_{2}$ has values

$$
G_{2}(x, \xi)= \begin{cases}\int_{x}^{\xi}[1 / r(z)] d z / \int_{a}^{\xi}[(z-a) / r(z)] d z, & a \leqq x \leqq \xi \leqq b, \\ 0, & a \leqq \xi \leqq x \leqq b .\end{cases}
$$

It is easily verified that $G_{2}$ is a convex function of $x$. Hence, it follows from (3.6) that 


$$
\lambda_{1}\left[G_{2}\right] \geqq \inf _{\xi} \lambda_{1}[q]
$$

where $q$ is defined by (3.7). But for each value of $\xi, q$ is sub- $(L)$ on $[a, b]$ as a function of $x$. This is a consequence of the fact that any increasing solution of $L(y)=0$ is concave and $q$ is convex. Furthermore, $q$ is increasing so that by Theorem 3.1 and (3.9) we have

$$
\lambda_{1}\left[G_{2}\right] \geqq \inf _{\xi} \lambda_{1}\left[G_{1}\right] \text {. }
$$

We now show that the infimum is actually attained for $\xi \in[a, b)$. We define the step function $H$ on $[a, b]$ with value $0(a \leqq x \leqq \xi)$ and value $G_{1}(b, \xi)(\xi<x \leqq b)$. Since $H(x, \xi) \geqq G_{1}(x, \xi)$, it follows from the comparison theorem for eigenvalues [7], p. 134, that $\lambda_{1}\left[G_{1}\right] \geqq \lambda_{1}[H]$ for each value of $\xi \in[a, b)$. Furthermore, $G_{1}$ is concave in $(\xi, b)$ and $\int_{\xi}^{b} G_{1}(x, \xi) d x=1$ so that $1 \leqq \int_{\xi}^{b} H(x, \xi) d x \leqq 2$. Now define $H_{1}$ on $[a, b]$ with values $H_{1}(x, \xi)=2 H(x, \xi) / \int_{\xi}^{b} H(x, \xi) d x$ so that $\int_{a}^{b} H_{1}(x, \xi) d x=2$ and $H_{1}(x, \xi) \geqq H(x, \xi)$. Again, by the comparison theorem for eigenvalues, $\lambda_{1}\left[H_{1}\right] \leqq \lambda_{1}[H] \leqq \lambda_{1}\left[G_{1}\right]$ for each value of $\xi \in[a, b)$. But $\lambda_{1}\left[H_{1}\right]$ may be computed explicitly and be shown to increase without bound as $\xi \rightarrow b$. Hence, the same is true of $\lambda_{1}\left[G_{1}\right]$. This and the continuity of $\lambda_{1}\left[G_{1}\right]$ as a function of $\xi$ on $[a, b)$ imply the existence of a number $\xi^{\prime} \in[a, b)$ such that

$$
\lambda_{1}[\rho] \int_{a}^{b} \rho(x) d x \geqq\left.\lambda_{1}\left[G_{1}\right]\right|_{\xi=\xi^{\prime}}=\lambda_{0} .
$$

We now consider the $\inf _{\rho \in E_{2}} \lambda_{1}[\rho]$ where

$$
E_{2}=\left\{\rho \mid \rho \text { is } \operatorname{super-}(L) \text { on }[a, b], \int_{a}^{b} \rho(x) d x=M\right\} .
$$

Let $N(\xi)=\int_{a}^{b} y_{0}(x) g(x, \xi) d \xi$ where $g(x, \xi)$ is defined by (2.8). We may thus define ${ }_{a}$ on the set $\{(x, \xi) \mid a \leqq x \leqq b, a \leqq \xi \leqq b\}$ with values determined by

$$
G(x, \xi)=y_{0}(x) g(x, \xi) / N(\xi)
$$

on $\{(x, y) \mid a \leqq x \leqq b, a<\xi<b\}$ and $G(x, a)=\lim _{\xi \rightarrow a} G(x, \xi), G(x, b)=$ $\lim _{\xi \rightarrow b} G(x, \xi)$. It may be shown by Reid's lemma that $G$ is a super- $(L)$ function of $x$ on $[a, b]$. In particular, $L(G)=0$ except at $x=\xi$. We also note that $\int_{a}^{b} G(x, \xi) d x=1$.

THEOREM 3.3 Let $\lambda_{1}[\rho]$ be the lowest eigenvalue of a string of length $(b-a)$ with elastically held ends and density $\rho(x), x \in[a, b]$. If $\rho$ is a super- $(L)$ function on $[a, b]$ where $L(y)$ is defined by (1.2), 
then

$$
\lambda_{1}[\rho] \int_{a}^{b} \rho(x) d x \geqq \min _{\xi \in[a, b]} \lambda_{1}[G]
$$

with $G$ defined by (3.10).

Proof. We first show that $\rho$ has an integral representation of the form

$$
\rho(x)=\int_{a}^{b} G(x, \xi) N(\xi) d h(\xi)
$$

where $G(x, \xi)$ is defined by (3.10) and $h$ is increasing. By Theorem 2.2 and (3.10), we have

$$
\begin{aligned}
\rho(x)= & \int_{a}^{b} G(x, \xi) N(\xi) d f(\xi) \\
& +\left[y_{0}(x) \int_{a}^{b} \eta_{0}(z) d z\right]\left[\rho(a) \int_{x}^{b} \eta_{0}(z) d z / y_{0}(a)+\rho(b) \int_{a}^{x} \eta_{0}(z) d z / y_{0}(b)\right] .
\end{aligned}
$$

But $N(\xi)$ may be written in the form

$$
N(\xi)=\left[\int_{a}^{\xi} \eta_{0}(z) d z \int_{\xi}^{b} \eta_{0}(z) Y_{0}(z) d z+\int_{\xi}^{b} \eta_{0}(z) d z \int_{a}^{\xi} \eta_{0}(z) Y_{0}(z) d z\right] / \int_{a}^{b} \eta_{0}(z) d z
$$

where $Y_{0}(x)=\int_{a}^{x} y_{0}(z) d z$. From this it may be seen that

$$
G(x, a)=\lim _{\xi \rightarrow a} G(x, \xi)=y_{0}(x) \int_{x}^{b} \eta_{0}(z) d z / \int_{a}^{b} \eta_{0}(z)\left[Y_{0}(z)-Y_{0}(a)\right] d z
$$

and

$$
G(x, b)=\lim _{\xi \rightarrow b} G(x, \xi)=y_{0}(x) \int_{a}^{x} \eta_{0}(z) d z / \int_{a}^{b} \eta_{0}(z)\left[Y_{0}(b)-Y_{0}(z)\right] d z .
$$

Hence, the last term on the right of (3.12) may be written in the form

$$
\begin{aligned}
G(x, a) \rho(a) \int_{a}^{b} \eta_{0}(z) Y_{0}(z) d z / y_{0}(a) \int_{a}^{b} \eta_{0}(z) d z \\
\quad+G(x, b) \rho(b) \int_{a}^{b} \eta_{0}(z)\left[Y_{0}(a)-Y_{0}(z)\right] d z / y_{0}(b) \int_{a}^{b} \eta_{0}(z) d z \\
=\int_{a}^{b} G(x, \xi) d g(\xi)
\end{aligned}
$$

where $g(\xi)$ is a step function with the appropriate jumps at $a$ and $b$. Setting $h(\xi)=f(\xi)+g(\xi)$, we get (3.11).

By Lemma 3.1, we have

$$
\lambda_{1}[\rho] \int_{a}^{b} \rho(x) d x \geqq \inf _{\xi \in[a, b]} G(x, \xi) .
$$


That this lower bound is actually attained for some $\xi^{\prime} \in[a, b]$ follows from the fact that $G(x, \xi)$ and hence $\lambda_{1}[G]$ is a continuous function of $\xi$ on $[a, b]$.

We apply our result to the case where $L(y)=y^{\prime \prime}+y, x \in[-\pi / 4, \pi / 4]$ and $u(\pi / 4)=u(-\pi / 4)=0$. It is readily verified that in this case

$$
G(x, \xi)= \begin{cases}\frac{(\cos \xi-\sin \xi)(\cos x+\sin x)}{2 \sqrt{2}(\cos \xi-\sin \pi / 4)}, & -\pi / 4 \leqq x \leqq \xi \leqq \pi / 4, \\ \frac{(\cos \xi+\sin \xi)(\cos x-\sin x)}{2 \sqrt{2}(\cos \xi-\sin \pi / 4)}, & -\pi / 4 \leqq \xi \leqq x \leqq \pi / 4 .\end{cases}
$$

By Theorem 3.3,

$$
\lambda_{1}[\rho] \int_{-\pi / 4}^{x / 4} \rho(x) d x \geqq \min _{\xi} \lambda_{1}[G] .
$$

We show that the minimizing value of $\xi$ is $\xi=0$.

Consider the set $E(y)=\{x \mid G(x, \xi) \geqq y\}$ and denote the measure of that set by $\mu(E(y))$. Setting $M(y)=1 / 2 \mu(E(y))$, we define the symmetrization of $G(x, \xi)$ to be

$$
\Gamma(x, \xi)= \begin{cases}M^{-1}(x), & 0 \leqq x \leqq \pi / 4, \\ \Gamma(-x, \xi) & -\pi / 4 \leqq x \leqq 0 .\end{cases}
$$

It is shown in [5] that this symmetrization decreases the lowest eigenvalue of a string with density $G(x, \xi)$, i.e., $\lambda_{1}[G] \geqq \lambda_{1}[\Gamma]$ for each value of $\xi \in[-\pi / 4, \pi / 4]$ and for the boundary conditions $u(-\pi / 4)=$ $u(\pi / 4)=0$. Carrying out this symmetrization, we find

$$
\Gamma(x, \xi)= \begin{cases}\frac{\cos 2 \xi \cos 2 x}{2(\sqrt{2} \cos \xi-1) \sqrt{(1}+\cos 2 \xi \sin 2 x)}, & 0 \leqq x \leqq \pi / 4, \\ \frac{\cos 2 \xi \cos 2 x}{2(\sqrt{2} \cos \xi-1) \sqrt{(1}-\cos 2 \xi \sin 2 x)}, & -\pi / 4 \leqq x \leqq 0 .\end{cases}
$$

Furthermore, in each of the intervals $(0, \pi / 4)$ and $(-\pi / 4,0)$, straight forward computations yield

$$
\frac{\partial^{2} \Gamma}{\partial x^{2}}+\Gamma \leqq 0
$$

so that $\Gamma$ is super- $(L)$ in each interval (see[11]). Also $\partial \Gamma /\left.\partial x\right|_{x=0-}>0$ and $\partial \Gamma /\left.\partial x\right|_{x=0++}<0$ so that $\Gamma$ is super- $(L)$ for $x \in(-\pi / 4, \pi / 4)$. Since $\Gamma(x, \xi)=G(x, \xi)$ if and only if $\xi=0$, we may conclude that (3.13) holds with $\xi=0$.

Finally, we remark that the results described in this example can be extended to any interval whose length is less than $\pi$.

We close this section with some results about the eigenvalues of 
the Sturm-Liouville system

$$
\begin{aligned}
\left(R(x) u^{\prime}\right)^{\prime}-[Q(x)+\lambda P(x)] u & =0, \\
u^{\prime}(a)-h_{a} u(a)=0, u^{\prime}(b)+h_{b} u(b) & =0,\left(h_{a}, h_{b}>0\right),
\end{aligned}
$$

where $P$ and $Q$ are nonnegative integrable functions on $[a, b]$ and $R \in C^{\prime}[a, b]$ is positive. Since the lowest eigenvalue of this system is given by $\lambda_{1}=\min _{v} R[v]$ where the minimum is over all functions $v \in C^{\prime}[a, b]$ and $R(v)$ is the Rayleigh quotient ([7], p. 126)

$$
R[v]=\frac{\int_{a}^{b}\left(R v^{\prime 2}+Q v^{2}\right) d x+R(a) h_{1} v^{2}(a)+R(b) h_{2} v^{2}(b)}{\int_{a}^{b} P v^{2} d x}
$$

the results of Theorems 3.1 and 3.3 may be generalized immediately to include this case. Furthermore, the following theorem can be proved (see [1], p. 462).

THEOREM 3.4. Let $\lambda_{1}[Q]$ denote the lowest eigenvalue of the SturmLiouville system (3.14) and let $k=\int_{a}^{b} Q(x) d x$. If $Q$ is sub- $(L)$ on $[a, b]$, then

$$
\lambda_{1}[Q] \geqq \min \left\{\inf _{\xi} \lambda_{1}\left[k G_{1}\right], \inf _{\xi} \lambda_{1}\left[k G_{2}\right]\right\}
$$

where $G_{1}(x, \xi)$ and $G_{2}(x, \xi)$ are defined by (3.1) and (3.2) respectively. If $Q$ is super- $(L)$ on $[a, b]$, then

$$
\lambda_{1}[Q] \geqq \inf _{\xi} \lambda_{1}[k G]
$$

where $G(x, \xi)$ is defined by (3.10).

4. Lower bounds for the higher eigenvalues. We now look for lower bounds of $\lambda_{n}[\rho]$, the $n$th eigenvalue of the system (1.1), when $\rho \in E_{1}$,

$$
E_{1}=\left\{\rho \mid \text { is } \operatorname{sub}-(L) \text { on }[a, b], \int_{a}^{b} \rho(x) d x=M\right\} .
$$

THEOREM 4.1. Let $\lambda_{n}[\rho]$ be the nth eigenvalue of a string of length $(b-a)$ with elastically held ends and density $\rho(x)$, where $\rho$ is a sub- $(L)$ function on $[a, b]$. Let $G_{1}(x, \xi)$ and $G_{2}(x, \xi)$ be defined by (3.1) and (3.2) respectively. Then

$$
\lambda_{n}[\rho] \int_{a}^{b} \rho(x) d x \geqq \inf _{\xi_{1} \cdots, \xi_{n}} \lambda_{n}\left[\sum_{0}^{m} \alpha_{k} G_{1}\left(x, \xi_{k}\right)+\sum_{m+1}^{n+1} \alpha_{k} G_{2}\left(x, \xi_{k}\right)\right]
$$

where $\alpha_{k} \geqq 0$ and $\sum_{1}^{n} \alpha_{k}=1$. Furthermore, the $\xi_{k}$ 's are related by 
the inequalities, $a=\xi_{0}<\xi_{m+1}<\xi_{m+2}<\cdots<\xi_{n}<\xi_{1}<\xi_{2}<\cdots<\xi_{m}<\xi_{n+1}$.

Proof. Let $u_{n}(x)$ be the $n$th eigenfunction of the system (1.1) corresponding to the eigenvalue $\lambda_{n}[\rho]$. This function is known to have exactly $n-1$ zeros in $(a, b)$. Furthermore, $u_{n}^{2}(x)$ will have exactly $n$ maximum points in $[a, b]$. A maximum can occur at $a$ if and only if $u_{n}^{\prime}(a)=0$ and at $b$ if and only if $u_{n}^{\prime}(b)=0$, since $u_{n}$ is concave in $[a, b]$. Let $x_{2 k}(k=1, \cdots, n-1)$ denote the zeros and $x_{2 k-1}(k=1, \cdots, n)$ denote the maximum points of $u_{n}^{2}(x)$. Let $x_{0}=\alpha$, $x_{2 n}=b$ and note that if $a$ is a maximum point, then $x_{0}=x_{1}$, and if $b$ is a maximum point, then $x_{2 n-1}=x_{2 n}$.

We assume for the present that there exists a function $q$ such that

$$
\int_{x_{2 k}}^{x_{2(k+1)}} q(x) u_{n}^{2}(x) d x \geqq \int_{x_{2 k}}^{x_{2(k+1)}} \rho(x) u_{n}^{2}(x) d x \quad(k=0, \cdots, n-1) .
$$

In each of the intervals $\left(x_{2 k}, x_{2(k+1)}\right)$, it is well known ([7], p. 69) that

$$
\lambda_{n}[\rho]=\frac{\int_{x_{2 k}}^{x_{2(k+1)}} u_{n}^{\prime 2}(x) d x}{\int_{x_{2 k}}^{x_{2(k+1)}} \rho(x) u_{n}^{2}(x) d x}=R_{k}\left[\rho, u_{n}\right], \quad(k=1,2, \cdots, n-2) .
$$

In $\left(a, x_{2}\right)$, we have

$$
\lambda_{n}[\rho]=\frac{\int_{a}^{x_{2}} u_{n}^{\prime 2}(x) d x+h_{a} u_{n}^{2}(a)}{\int_{a}^{x_{2}} \rho(x) u_{n}^{2}(x) d x}=R_{1}\left\lfloor\rho, u_{n}\right\rfloor
$$

and in $\left(x_{2 n-2}, b\right)$ we have

$$
\lambda_{n}[\rho]=\frac{\int_{x_{2 n-2}}^{b} u_{n}^{\prime 2}(x) d x+h_{b} u_{n}^{2}(b)}{\int_{x_{2 n-2}}^{b} \rho(x) u_{n}^{2}(x) d x}=R_{n}\left[\rho, u_{n}\right]
$$

$h_{a}, h_{b}<\infty$. If $h_{a}=h_{b}=\infty$, the last term in the numerators of $R_{1}\left[\rho, u_{n}\right]$ and $R_{n}\left[\rho, u_{n}\right]$ does not occur. By (4.1) it follows that

$$
\lambda_{n}[\rho] \geqq R_{k}\left[q, u_{n}\right] \geqq \min _{v} R_{k}[q, v]
$$

$(k=1,2, \cdots, n)$ where the minimum for each $k$ is taken over only those functions $v$ which satisfy the same boundary conditions as $u_{n}(x)$. In particular

$$
\lambda_{n}[\rho] \geqq \max _{k}\left\{\min _{v} R_{k}[q, v]\right\} .
$$

But the quantity on the right is greater than the $n$th eigenvalue 
$\lambda_{n}[q]$ of a string with density $q(x)$ and satisfying the boundary conditions of the system (1.1).

We now show that for every $\rho(x), x \in[a, b]$ there is a function $q(x)$ of the form

$$
q(x)=\sum_{k=0}^{m} \alpha_{k} G_{1}\left(x, \xi_{k}\right)+\sum_{k=m+1}^{n+1} \alpha_{k} G_{2}\left(x, \xi_{k}\right), x \in[a, b]
$$

such that (4.1) is satisfied. As in the statement of the theorem $\xi_{0}=a$ and $\xi_{n+1}=b$ while $a<\xi_{m+1}<\xi_{m+2}<\cdots<\xi_{n} \leqq \xi_{1}<\xi_{2}<\cdots<\xi_{m}<b$. Consider the integrals $\int_{x_{k-1}}^{x_{k}} \rho(x) \mathrm{d} x,(k=1,2, \cdots, 2 n)$. Using the change of variables (2.1) of Reid's lemma, we get

$$
\int_{x_{k-1}}^{x_{k}} \rho(x) d x=\int_{t_{k-1}}^{t_{k}} \rho^{*}(t) w(t) d t
$$

$(k=1,2, \cdots, 2 n)$ where $w(t)=y_{0}^{3}(x(t)) r(x(t))$ and $x(\alpha)=a, x\left(t_{k}\right)=x_{k}$ $(k=1,2, \cdots, 2 n-1), x(\beta)=b$. It is possible to show that for every nonnegative function $\rho^{*}$ which is convex on $(\alpha, \beta)$, there is a continuous piecewise linear nonnegative convex function $q^{*}$ defined on $[\alpha, \beta]$ such that

$$
\int_{t_{k-1}}^{t_{k}} \rho^{*}(t) w(t) d t=\int_{t_{k-1}}^{t_{k}} q^{*}(t) w(t) d t
$$

$(k=0,1, \cdots, 2 n-1)$. Furthermore, either $q^{*}$ is linear except at the points $\tau_{k}(k=1, \cdots, n-1)$ where $t_{2 k-1}<\tau_{k}<t_{2 k+1}$ or this is true except for some value of $k$ where we might have $t_{2 k-1}<\tau_{k}<\tau_{k}^{\prime}<t_{2 k+1}$ with $q^{*}(t) \equiv 0$ for $t \in\left[\tau_{k}, \tau_{k}^{\prime}\right]$. The derivation of this result is given in [1], p. 456, with $w(t) \equiv 1$. The proof given there holds for this more general result if $w(t)$ is inserted in the integrals which occur there.

We then define $q(x)=y_{0}(x) q^{*}\left(\int_{x_{0}}^{x} \eta_{0}(z) d z\right), x \in[a, b]$. Since $q^{*}(t)$ is piecewise linear, we have $L(q)=0^{0}$ except at the points $\xi_{k}=x\left(\tau_{k}\right)$ where $\tau_{k}$ is defined above. By Reid's lemma, $q(x)$ is sub- $(L)$ in $[a, b]$ and furthermore, the change of variables (2.1) and (4.2) yield

$$
\int_{x_{k}}^{x_{k+1}} \rho(x) d x=\int_{x_{k}}^{x_{k+1}} q(x) d x .
$$

The above exceptional points $\xi_{k}$ are such that $q\left(\xi_{k}\right) \leqq \rho\left(\xi_{k}\right)$. This follows immediately from $q^{*}\left(\tau_{k}\right) \leqq \rho^{*}\left(\tau_{k}\right)$ which in turn is a consequence of the piecewise linearity of $q^{*}(t)$ and the convexity of $q^{*}(t)$ and $\rho^{*}(t)$.

We now note that in the interval $\left(x_{2 k}, x_{2 k+1}\right),(k=0,1, \cdots, n-1)$, there exists a number $\alpha_{k}$ such that $\rho(x) \geqq q(x), x \in\left(x_{k}, \alpha_{k}\right)$ and $q(x) \leqq q(x), x \in\left(\alpha_{k}, x_{2 k+1}\right)$. Also in $\left(x_{2 k+1}, x_{2 k+2}\right), \quad(k=0,1, \cdots, n-1)$, 
there exists a $\beta_{k} \in\left(x_{2 k+1}, x_{2 k+2}\right)$ such that $\rho(x) \leqq q(x), x \in\left(x_{2 k+1}, \beta_{k}\right)$ and $\rho(x) \geqq q(x), x \in\left(\beta_{k}, x_{k+1}\right)$. If $\rho \equiv q$, this is trivially true. Otherwise in $\left(\xi_{k}, \xi_{k+1}\right), L(q)=0$ and $\rho$ is sub- $(L)$. Hence, they have at most two common values there since otherwise they are identical. But (4.3) implies that $\rho$ and $q$ must have at least one common value in $\left(x_{2 k}, x_{2 k+1}\right)$ and at least one common value in $\left(x_{2 k+1}, x_{2 k+2}\right)(k=0,1, \cdots, n)$. Thus $\rho \equiv q$ or the $\alpha_{k}$ and $\beta_{k}$ defined above are uniquely determined. By Lemma 3.2, we then find that

$$
\int_{x_{k-1}}^{x_{k}} \rho u_{n}^{2} d x \leqq \int_{x_{k-1}}^{x_{k}} q u_{n}^{2} d x
$$

$(k=1,2, \cdots, 2 n)$. On summing these we get the desired inequality (4.1).

It remains to express $q(x)$ in the form given in the theorem. This follows easily from the integral representation given in Theorem 2.1 .

We now look for the $\inf _{\rho \in E_{2}} \lambda_{n}[\rho]$, where

$$
E_{2}=\left\{\rho \mid \rho \text { is super- }(L) \text { on }[a, b], \int_{a}^{b} \rho(x) d x=M\right\} .
$$

THEOREM 4.2. Let $\lambda_{n}[\rho]$ be the nth eigenvalue of a string of length $(b-a)$ with elastically held ends and density $\rho(x)$, where $\rho(x)$ is super- $(L)$ on $[a, b]$. Let $G(x, \xi)$ be defined by (3.10). Then

$$
\lambda_{n}[\rho] \int_{a}^{b} \rho(x) d x \geqq \inf _{\xi_{1}, \ldots, \xi_{n} \in[a, b]} \lambda_{n}\left[\sum_{k=1}^{n} \alpha_{k} G\left(x, \xi_{k}\right)\right]
$$

with $\sum_{k=1}^{n} \alpha_{k}=1$.

Proof. As in the proof of Theorem 4.1, we denote the maximum points of $u_{n}^{2}$ by $x_{2 k-1}(k=1, \cdots, n)$ and the minimum points or zeros by $x_{2 k}(k=0,1, \cdots, n)$. We let $x_{0}=a$ and $x_{2 n}=b$. The existence of a function $q$ on $[a, b]$ such that

$$
\int_{x_{k-1}}^{x_{k}} q u_{n}^{2} d x \geqq \int_{x_{k-1}}^{x_{k}} \rho u_{n}^{2} d x \quad(k=1,2, \cdots, 2 n)
$$

implies, as shown in the proof of Theorem 4.1, that

$$
\lambda_{n}[q] \leqq \lambda_{n}[\rho] \text {. }
$$

Thus we need only show that for every super- $(L)$ function $\rho$ on $[a, b]$ there is a function $q$ on $[a, b]$ with values $q(x)=\sum_{k=1}^{n} \alpha_{k} G\left(x, \xi_{k}\right)$ where $G(x, \xi)$ is defined by (3.10) such that (4.4) holds. The conclusion of our theorem then follows.

As in the proof of the previous theorem we make the change of variables (2.1) of Ried's lemma to get 


$$
\int_{x_{k-1}}^{x_{k}} \rho(x) d x=\int_{t_{k-1}}^{t_{k}} \rho^{*}(t) w(t) d t
$$

$(k=1,2, \cdots, 2 n)$ where $w(t)=y_{0}^{3}(x(t)) r(x(t))$.

It is possible to show that for every nonnegative function $\rho^{*}$ which is concave on $(\alpha, \beta)$, there is a continuous piecewise linear nonnegative concave function $q^{*}$ defined on $(\alpha, \beta)$ such that

$$
\int_{t_{k-1}}^{t_{k}} \rho^{*}(t) w(t) d t=\int_{t_{k-1}}^{t_{k}} q^{*}(t) w(t) d t, \quad(k=1,2, \cdots, 2 n) .
$$

Furthermore, there are at most $n$ values $\tau_{1}<\tau_{2}<\cdots<\tau_{n}$ where $q^{*}$ is piecewise linear and $t_{2 k-2}<\tau_{k}<t_{2 k}(k=1, \cdots, n)$. This result is derived in [1], p. 461, when $w(t) \equiv 1$. The derivation given there is also valid for this case if the weight function $w$ is introduced in the integrals occurring there.

We now define

$$
q(x)=y_{0}(x) q^{*}\left(\int_{x_{0}}^{x} \eta_{0} d z\right), \quad x \in[a, b] .
$$

Since $q^{*}$ is linear, it follows that $L(q)=0$ except at the points $\xi_{k}=x\left(\tau_{k}\right)$. By Reid's Lemma, $q$ is super- $(L)$ on $[a, b]$ and furthermore, (4.5), (4.6) and (2.1) yield

$$
\int_{x_{k-1}}^{x_{k}} \rho(x) d x=\int_{x_{k-1}}^{x_{k}} q(x) d x, \quad(k=1,2, \cdots, 2 n) .
$$

We note that

$$
\int_{x_{k-1}}^{x_{k}} \rho u_{n}^{2} d x=\int_{x_{k-1}}^{x_{k}}(-\rho)\left(-u_{n}^{2}\right) d x
$$

and

$$
\int_{x_{k-1}}^{x_{k}} q u_{n}^{2} d x=\int_{x_{k-1}}^{x_{k}}(-q)\left(-u_{n}^{2}\right) d x
$$

It then follows, as in the proof of Theorem 4.1, that

$$
\int_{x_{k-1}}^{x_{k}}(-\rho)\left(-u_{n}^{2}\right) d x \leqq \int_{x_{k-1}}^{x_{k}}(-q)\left(-u_{n}^{2}\right) d x, \quad(k=1, \cdots, 2 n)
$$

and hence that

$$
\int_{x_{2 k-2}}^{x_{2 k}} \rho u_{n}^{2} d x \leqq \int_{x_{2 k-2}}^{x_{2 k}} q u_{n}^{2} d x, \quad(k=1, \cdots, n) .
$$

Moreover, $q(x)$ is easily represented in the form given in the theorem by use of the integral representation of a concave function given in. Theorem 2.2. 
5. Upper bounds for $\lambda_{n}[\rho]$. In this section we prove the following two theorems where $\lambda_{n}[\rho]$ is the $n$th eigenvalue of (1.1). We exclude the case where the boundary conditions are $u^{\prime}(a)=u^{\prime}(b)=0$.

THEOREM 5.1. Let $\lambda_{n}[\rho]$ be the nth eigenvalue of a string of length $(b-a)$ with elastically held ends and with a bounded density $\rho(x)$ which is sub- $(L)$ on $[a, b]$. Then

$$
\lambda_{n}[\rho] \leqq \lambda_{n}\left[\rho_{1}\right]
$$

where $\rho_{1}$ is a bounded sub- $(L)$ function on $[a, b]$ such that $L\left(\rho_{1}\right)=0$ with the exception of at most $n+1$ points in $[a, b]$. Furthermore, $\int_{a}^{b} \rho(x) d x=\int_{a}^{b} \rho_{1}(x) d x$.

THEOREM 5.2. Let $\lambda_{n}[\rho]$ be the nth eigenvalue of a string held elastically at $x=a$ and $x=b$. If its density $\rho(x)$ is super- $(L)$ on $[a, b]$, then

$$
\lambda_{n[\rho]} \leqq \lambda_{n}\left[\rho_{2}\right]
$$

where $\rho_{2}$ is a super- $(L)$ function on $[a, b]$ such that $L\left(\rho_{2}\right)=0$ with the exception of at most $n$ points in $(a, b)$. Furthermore, $\int_{a}^{b} \rho(x) d x=$ $\int_{a}^{b} \rho_{2}(x) d x$.

We remark that by Theorem 2.1, $\rho_{1}(x)$ may be expressed as a linear combination of $G_{1}\left(x, \xi_{k}\right)$ defined by (3.1) and $G_{2}\left(x, \xi_{k}\right)$ defined by (3.2) for appropriate values of $\xi_{k}(k=1, \cdots, n+1)$. By Theorem 2.2, $\rho_{2}(x)$ may be expressed as a linear combination of $G\left(x, \xi_{k}\right)$ defined by (3.10) for appropriate values of $\xi_{k}(k=1, \cdots, n)$.

The proofs of Theorems 5.1 and 5.2 will be based on a result due to M. G. Krein [10] which we state as

LEMMA 5.1. Consider the set of functions $E=\left\{\rho \mid \int_{a}^{b} \rho(x) d x=M\right.$, $0 \leqq \rho(x) \leqq H\}$. Let $\mu$ be a cumulation point of $\left\{\lambda_{n}[\rho] \mid \rho \in E\right\}$ and let $\left\{\rho_{\nu}\right\}$ be any sequence contained in $E$ such that $\left\{\lambda_{n}\left[\rho_{\nu}\right]\right\} \rightarrow \mu$. Then there is a function $\rho_{0} \in E$ such that for some subsequence of $\left\{\rho_{\nu}\right\}$ we have $\left\{\int_{a}^{x} \rho_{\nu_{k}}(z) d z\right\} \rightarrow \int_{a}^{x} \rho_{0}(z) d z$ uniformly in $x$ and $\lambda_{n}\left[\rho_{0}\right]=\mu$.

Krein's proof was carried out for the eigenvalue problem with boundary conditions $u(a)=u(b)=0$. However, his proof is also valid for the more general boundary conditions of (1.1) if we exclude the free endpoint problem $u^{\prime}(a)=u^{\prime}(b)=0$ from consideration. For all that is required is that the Green's function of $u^{\prime \prime}=0$ with the boundary conditions of (1.1) together with its partial derivative with 
respect to $x$ be bounded. It is not difficult to see that this is the case (see [7], p. 83).

We also make use of

LEMMA 5.2. The first variation of $\lambda_{n}[\rho]$ of the system (1.1) under the restriction $\int_{a}^{b} \rho(x) d x=M$ is

$$
\delta \lambda_{n}[\rho]=-\lambda_{n}[\rho] \int_{a}^{b} \delta \rho(x) u_{n}^{2}(x) d x
$$

where $u_{n}$ is the normalized eigenfunction corresponding to $\lambda_{n}[\rho]$ and $\int_{a}^{b}(\delta \rho(x)) d x=0$.

A proof of this is given in [2], p. 1186, for the boundary conditions $u(a)=u(b)=0$. The same proof holds without change for the boundary conditions used here.

We now give a proof of Theorem 5.1. Let $E_{1}$ denote the set of functions

$$
E_{1}=\left\{\rho \mid \int_{a}^{b} \rho(x) d x=M, 0 \leqq \rho(x) \leqq H, \rho \text { is } \operatorname{sub}-(L) \text { on }[a, b]\right\} .
$$

By the comparison theorem for eigenvalues, $\lambda_{1}[\rho] \leqq \lambda_{1}[H]$ for every $\rho \in E_{1}$. Hence, $\sup _{E_{1}} \lambda_{1}[\rho]=\mu$ is finite. Choose a sequence $\left\{\rho_{\nu}\right\}$ from $E_{1}$ such that $\left\{\lambda_{n}\left[\rho_{\nu}\right]\right\} \rightarrow \mu$. By Lemma 5.1, there is a subsequence such that $\mu=\lambda_{n}\left[\rho_{0}\right]$ and $\left\{\int_{a}^{x} \rho_{\nu_{k}}(z) d z\right\} \rightarrow \int_{a}^{x} \rho_{0}(z) d z$ where $\rho_{0} \in E_{1}$. We drop the subscript $k$ since no confusion can result. We now show that $\rho_{0} \in E_{1}$ also. By the change of variables (2.1) of Reid's lemma we have

$$
\int_{a}^{x} \rho_{\nu}(z) d z=\int_{a}^{t} \rho_{\nu}^{*}(\zeta) w(\zeta) d \zeta
$$

where $w(\zeta)=y_{0}^{3}(x(\zeta)) r(x(\zeta)), \quad \rho_{\nu}^{*}(\zeta)=\rho(x(\zeta)) / y_{0}(x(\zeta)), \quad \zeta \in[\alpha, \beta] . \quad$ The above subsequence then converges to

$$
\int_{a}^{t} \rho_{0}(x(\zeta)) w(\zeta) d \zeta
$$

By Reid's lemma $\rho_{\nu}^{*}$ is a convex function for $t \in(\alpha, \beta)$. Also $0 \leqq$ $\rho_{\nu}^{*} \leqq H$ so that for any $t \in[\alpha+\delta, \beta-\delta]$ we have

$$
\left|\left[\rho_{\nu}^{*}(t+h)-\rho_{\nu}^{*}(t)\right] / h\right|<H / \delta
$$

for all $\nu$. Hence $\left\{\rho_{\nu}^{*}\right\}$ is an equicontinuous family of functions on $[\alpha+\delta, \beta-\delta]$ and therefore contains a uniformly convergent subsequence which converges uniformly to a continuous function $\rho^{*}$, $t \in[\alpha+\delta, \beta-\delta]$. This implies that 


$$
\lim _{\nu \rightarrow \infty} \int_{\alpha+\delta}^{t} \rho_{\nu}^{*}(\zeta) w(\zeta) d \zeta=\int_{\alpha+\delta}^{t} \rho^{*}(\zeta) w(\zeta) d \zeta=\int_{\alpha+\delta}^{t} \rho_{0}(x(\zeta)) w(\zeta) d \zeta
$$

and hence $\rho^{*}(t)=\rho_{0}(x(t)) / y_{0}(x(t))$ for all $t \in(\alpha, \beta)$. Furthermore, since $\rho_{\nu}^{*}(t)$ is convex and bounded, so is $\rho_{0}(x(t))$. By Reid's lemma, $\rho_{0}(x)$ is then bounded by $H$ and is $\operatorname{sub}-(L)$ on $[a, b]$. By Lemma 5.1 we also have $\lambda_{n}\left[\rho_{\nu}\right] \rightarrow \lambda_{n}\left[\rho_{0}\right]$.

The above result is equivalent to the statement that $\max _{\rho \in E_{1}} \lambda_{n}[\rho]=$ $\lambda_{n}\left[\rho_{1}\right]$ where $\rho_{1} \in E_{1}$. We show that this function $\rho_{1}$ is as described in Theorem 5.1. By Lemma 5.2, we must have

$$
\delta \lambda_{n}\left[\rho_{1}\right]=-\lambda_{n}\left[\rho_{1}\right] \int_{a}^{b} \delta \rho_{1} u_{n}^{2} d x \leqq 0 .
$$

We will show that $L\left(\rho_{1}\right)=0$ except at most $(n+1)$ points in $[a, b]$ or there is a function $q(x) \in[a, b]$ such that $\delta \lambda_{n}\left[\rho_{1}\right]>0$ where $\delta \rho_{1}=$ $\varepsilon q$ and $\rho_{1}+\delta \rho_{1} \in E_{1}$. The latter case is a contradiction. Thus the theorem will be proved.

Assume that $\rho_{1}$ is not of the form described above. As in $\S 4$, we denote the maximum points of $u_{n}^{2}$ by $x_{2 k-1}(k=1,2, \cdots, n)$ and the minimum points by $x_{2 k}(k=0,1,2, \cdots, n)$. The change of variables (2.1) of Reid's lemma yields

$$
\int_{x_{k-1}}^{x_{k}} \rho_{1}(x) d x=\int_{t_{k-1}}^{t_{k}} \rho_{1}^{*}(t) w(t) d t, \quad(k=1,2, \cdots, 2 n),
$$

where $\rho_{1}^{*}(t)=\rho_{1}(x(t)) / y_{0}(x(t))$ is convex and $w(t)=y_{0}^{3}(x(t)) r(x(t))$. In [2], p. 1193, it was shown that a function $s^{*}$ on $[\alpha, \beta]$ exists such that

$$
\int_{t_{k-1}}^{t_{k}} s^{*}(t) w(t) d t=\int_{t_{k-1}}^{t_{k}} \rho_{I}^{*}(t) w(t) d t
$$

$(k=1,2, \cdots, 2 n)$ where $s^{*}$ is convex on $[\alpha, \beta]$ and $\left(s^{*}\right)^{\prime \prime}=0$ with the exception of at most $n$ points $\tau_{k} \in\left(t_{2 k-2}, t_{2 k}\right)(k=1,2, \cdots, n)$ or $n+1$ points if $s^{*}$ vanishes in some sub-interval of $[\alpha, \beta]$. The proof as given there is for the case where $w(t) \equiv 1$; however, the more general result may be proved in exactly the same way. By Reid's lemma, (5.1) yields

$$
\int_{x_{k-1}}^{x_{k}} s(x) d x=\int_{x_{k-1}}^{x_{k}} \rho_{1}(x) d x
$$

where $s(x)=y_{0}(x) s^{*}\left(\int_{x_{0}}^{x} \eta_{0}(z) d z\right)$ is sub- $(L)$ on $[a, b]$ and $L\left(\rho_{1}\right)=0$ except at most $n$ or $n+1$ points $\xi_{k}=x\left(\tau_{k}\right)$ in $(a, b)$.

The function $s^{*}$ is defined in such a way that $s^{*}\left(\tau_{k}\right) \leqq \rho_{1}^{*}\left(\tau_{k}\right)$ $(k=1,2, \cdots, n)$. Hence it follows that $s\left(\xi_{k}\right) \leqq \rho_{1}\left(\xi_{k}\right)$. By the same argument used in the proof of Theorem 4.1, we may show that 


$$
\int_{x_{k-1}}^{x_{k}} s(x) u_{n}^{2}(x) d x<\int_{x_{k-1}}^{x_{k}} \rho_{1}(x) u_{n}^{2}(x) d x
$$

$(k=1,2, \cdots, 2 n)$. The inequality is strict since there is equality if and only if $s(x) \equiv \rho_{1}(x)$. Summing on $k$, we get

$$
\int_{a}^{b}\left[s(x)-\rho_{1}(x)\right] u_{n}^{2}(x) d x<0 .
$$

We let $q(x)=s(x)-\rho_{1}(x)$ and $\delta \rho_{1}(x)=\varepsilon q(x), \varepsilon>0$. Now $\rho_{1}(x)+\varepsilon q(x)=$ $(1-\varepsilon) \rho_{1}(x)+s(x)$ is sub- $(L)$ on $[a, b]$ as long as $\varepsilon \leqq 1$. Hence, we have by Lemma 5.2

$$
\delta \lambda_{n}\left[\rho_{1}\right]=-\lambda_{n}\left[\rho_{1}\right] \int_{a}^{b} \delta \rho_{1} u_{n}^{2} d x>0 .
$$

This proves Theorem 5.1.

Proof of Theorem 5.2. This proof follows the same general outline as the proof of Theorem 5.1. Let

$$
E_{2}=\left\{\rho \mid \int_{a}^{b} \rho(x) d x=M, \rho \text { is } \operatorname{super-}(L) \text { on }[a, b]\right\} .
$$

We first show that $\rho \in E_{2}$ implies that $|\rho(x)| \leqq H$ where $H$ depends only on $M$ and the operator $L(y)$. By Reid's lemma, we have

$$
\int_{a}^{b} \rho(x) d x=\int_{a}^{\beta} \rho^{*}(t) w(t) d t=M,
$$

where $\rho^{*}(t)=\rho(x(t)) / y_{0}(x(t))$ is concave on $[\alpha, \beta]$ and $w(t)=r(x(t)) y_{0}^{3}(x(t))$. Now $w(t)>0$ on $[\alpha, \beta]$ so that $\min _{t} w(t)>0$. Furthermore $\rho^{*}(t) \geqq 0$ so that it follows that $\int_{\alpha}^{\beta} \rho^{*}(t) d t \leqq M / \min w(t)$. This implies that $\rho^{*}(t) \leqq 2 M /(\beta-\alpha) \min w(\dot{t})$, for otherwise the convexity of $\rho^{*}$ would imply that $\int_{\alpha}^{\beta} \rho^{*}(t) d t>M / \min w(t)$. Hence by the definition of $\rho^{*}(t)$, we have $\rho(x) \leqq 2 M y_{0}(x) /(\beta-\alpha) \min w(t)=H$ for all $\rho \in E_{2}$.

By the same argument used in the proof of Theorem 5.1, we have

$$
\max _{\rho \in E_{2}} \lambda_{n}[\rho]=\lambda_{n}\left[\rho_{2}\right]
$$

where $\rho_{2} \in E_{2}$. Lemma 5.2 then implies that

$$
\delta \lambda_{n}\left[\rho_{2}\right]=-\lambda_{n}\left[\rho_{2}\right] \int_{a}^{b} \delta \rho_{2} u_{n}^{2} d x \leqq 0 .
$$

It remains to show that $\rho_{2}$ satisfied $L\left(\rho_{2}\right)=0$ with the exception of at most $n$ points in $[a, b]$ or there is a function $q$ on $[a, b]$ such that $\delta \lambda_{n}\left[\rho_{1}\right]>0$ where $\delta \rho_{2}=\varepsilon q$ and $\rho_{2}+\delta \rho_{2} \in E_{2}$. The latter alternative is a contradiction and hence our theorem will be proved. 
To show the existence of the function $q$ on $[a, b]$ if $\rho_{2}$ is not as described, we again rely on Reid's lemma and the corresponding result for concave functions given in [2], p. 1196. As in the previous proof, this leads to the result

$$
\int_{a}^{b} s(x) u_{n}^{2}(x) d x<\int_{a}^{b} \rho_{2}(x) u_{n}^{2}(x) d x
$$

where $s$ is a super- $(L)$ function on $[a, b]$ with $L(s)=0$ except at most $n$ points in $(a, b)$. The derivation of this inequality is so nearly the same as that used in Theorem 5.1, that we do not give the details here. We may now define $q(x)=s(x)-\rho_{2}(x)$ so that we have

$$
\begin{aligned}
\delta \lambda_{n}\left[\rho_{2}\right] & \left.=-\lambda_{1}\left[\rho_{2}\right]\right]_{a}^{b} \varepsilon q u_{n}^{2} d x \\
& \left.=\lambda_{1}\left[\rho_{2}\right]\right]_{a}^{b} \delta \rho_{2} u_{n}^{2} d x>0
\end{aligned}
$$

where $0<\varepsilon \leqq 1$ so that $\rho_{2}-\delta \rho_{2} \in E_{2}$.

6. Remarks. There are several results in [1] and [2] concerning bounds for eigenvalues when the density function is convex or concave which could be generalized to the cases discussed here. In particular, the results of $\S 2$ allow us to find lower bounds for the sum $\sum_{1}^{n}\left(1 / \lambda_{n}[\rho]\right)$ where $\rho$ is $\operatorname{sub}-(L)$ or $\operatorname{super}-(L)$ on $[a, b]$ and $\int_{a}^{b} \rho(x) d x=M$. (See [1], Theorem 3.2, Theorem 4.2.) Also, all of the results presented here lead to a corresponding result for a vibrating rod with clamped or hinged ends and even for more general boundary conditions.

We also note that the results concerning lower bounds may be obtained by use of the variational approach given in $\S 5$ when every $\rho$ is bounded.

\section{REFERENCES}

1. D. Banks, Bounds for the eigenvalues of some vibrating systems, Pacific J. Math., 10 (1960), 439-474.

2. U Upper bounds for the eigenvalues of some vibrating systems, Pacific J. Math., 11 (1961), 1183-1203.

3. E. F. Beckenbach, Generalized convex functions, Bull. Amer. Math. Soc., 43 (1937), 363-371.

4. P. R. Beesack, A note on an integral inequality, Proc. Amer. Math. Soc., 8 (1957), 875-879.

5. P. R. Beesack and B. Schwarz, On the zeros of solutions of second order linear differential equations, Canadian J. Math., 8 (1956), 504-515.

6. F. F. Bonsall, The characterization of generalized convex functions, Quart. J. Math., Oxford Ser. (2) 1, (1950), 100-111.

7. L. Collatz, Eigenwertprobleme und ihre numerische Behandlung, Chelsea, New York, 1948. 
8. R. Courant and D. Hilbert, Methods of mathematical physics, Interscience, New York, 1953.

9. J. Hersch, Propriétés de convexité du type de Weyle pour des problèmes de vibration ou d'équilibre, Z. Angew. Math. Phys., 12 (1961), 298-322.

10. M. G. Krein, On certain problems on the maximum and minimum of characteristic values and on Lyapunov zones of stability, Amer. Math. Soc. Trans., Ser. 2, 1 (1955), 163-187.

11. M. M. Peixoto, Generalized convex functions and second order differential inequalities, Bull. Amer. Math. Soc., 55 (1949), 563-572.

12. W. T. Reid, Variational aspects of generalized convex functions, Pacific J. Math., $\mathbf{9}$ (1959), 571-581.

13. B. Schwarz, On the extrema of the frequencies of non-homogeneous strings with equimeasurable density, J. of Math. and Mech., 10 (1961), 401-422.

UNIVERSITY OF CALIFORNIA, DAVIS 


\section{PACIFIC JOURNAL OF MATHEMATICS}

\section{EDITORS}

RalPh S. Phillips

Stanford University

Stanford, California

M. G. Arsove

University of Washington

Seattle 5 , Washington
J. Dugundu

University of Southern California

Los Angeles 7, California

Lowell J. Paige

University of California

Los Angeles 24, California

\section{ASSOCIATE EDITORS}
E. F. BECKENBACH
D. DERRY
H. L. ROYDEN
E. G. STRAUS
T. M. CHERRY
M. OHTSUKA
E. SPANIER
F. WOLF

\section{SUPPORTING INSTITUTIONS}

\author{
UNIVERSITY OF BRITISH COLUMBIA \\ CALIFORNIA INSTITUTE OF TECHNOLOGY \\ UNIVERSITY OF CALIFORNIA \\ MONTANA STATE UNIVERSITY \\ UNIVERSITY OF NEVADA \\ NEW MEXICO STATE UNIVERSITY \\ OREGON STATE UNIVERSITY \\ UNIVERSITY OF OREGON \\ OSAKA UNIVERSITY \\ UNIVERSITY OF SOUTHERN CALIFORNIA
}

\author{
STANFORD UNIVERSITY \\ UNIVERSITY OF TOKYO \\ UNIVERSITY OF UTAH \\ WASHINGTON STATE UNIVERSITY \\ UNIVERSITY OF WASHINGTON \\ AMERICAN MATHEMATICAL SOCIETY \\ CALIFORNIA RESEARCH CORPORATION \\ SPACE TECHNOLOGY LABORATORIES \\ NAVAL ORDNANCE TEST STATION
}

Mathematical papers intended for publication in the Pacific Journal of Mathematrcs should be typewritten (double spaced), and the author should keep a complete copy. Manuscripts may be sent to any one of the four editors. All other communications to the editors should be addressed to the managing editor, L. J. Paige at the University of California, Los Angeles 24, California.

50 reprints per author of each article are furnished free of charge; additional copses may be obtained at cost in multiples of 50 .

The Pacific Journal of Mathematics is published quarterly, in March, June, September, and December. Effective with Volume 13 the price per volume (4 numbers) is $\$ 18.00$; single issues, $\$ 5.00$. Special price for current issues to individual faculty members of supporting institutions and to individual members of the American Mathematical Society: $\$ 8.00$ per volume; single issues $\$ 2.50$. Back numbers are available.

Subscriptions, orders for back numbers, and changes of address should be sent to Pacific Journal of Mathematics, 103 Highland Boulevard, Berkeley 8, California.

Printed at Kokusai Bunken Insatsusha (International Academic Printing Co., Ltd.), No. 6 , 2-chome, Fujimi-cho, Chiyoda-ku, Tokyo, Japan.

\section{PUBLISHED BY PACIFIC JOURNAL OF MATHEMATICS, A NON-PROFIT CORPORATION}

The Supporting Institutions listed above contribute to the cost of publication of this Journal, but they are not owners or publishers and have no responsibility for its content or policies. 


\section{Pacific Journal of Mathematics}

\section{Vol. 13, No. $4 \quad$ June, 1963}

Dallas O. Banks, Bounds for eigenvalues and generalized convexity ........... 1031

Jerrold William Bebernes, A subfunction approach to a boundary value problem for

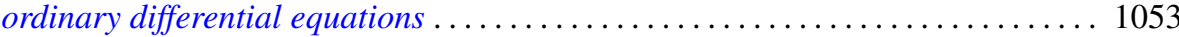

Woodrow Wilson Bledsoe and A. P. Morse, A topological measure construction . . . 1067

George Clements, Entropies of several sets of real valued functions . . . . . . . . . 1085

Sandra Barkdull Cleveland, Homomorphisms of non-commutative *-algebras . . . . . 1097

William John Andrew Culmer and William Ashton Harris, Convergent solutions of

ordinary linear homogeneous difference equations . . . . . . . . . . . . . . . 1111

Ralph DeMarr, Common fixed points for commuting contraction mappings . . . . . . 1139

James Robert Dorroh, Integral equations in normed abelian groups . . . . . . . . 1143

Adriano Mario Garsia, Entropy and singularity of infinite convolutions . . . . . . . 1159

J. J. Gergen, Francis G. Dressel and Wilbur Hallan Purcell, Jr., Convergence of extended Bernstein polynomials in the complex plane ................. 1171

Irving Leonard Glicksberg, A remark on analyticity of function algebras . . . . . . 1181

Charles John August Halberg, Jr., Semigroups of matrices defining linked operators

with different spectra ................................. 1187

Philip Hartman and Nelson Onuchic, On the asymptotic integration of ordinary

differential equations . . . . . . . . . . . . . . . . . . . . . . . . . . . . 1193

Isidore Heller, On a class of equivalent systems of linear inequalities . . . . . . . . . 1209

Joseph Hersch, The method of interior parallels applied to polygonal or multiply

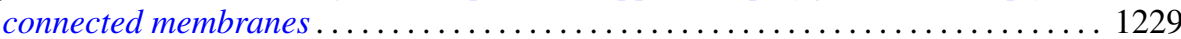

Hans F. Weinberger, An effectless cutting of a vibrating membrane . . . . . . . . . . 1239

Melvin F. Janowitz, Quantifiers and orthomodular lattices ....

Samuel Karlin and Albert Boris J. Novikoff, Generalized convex inequalities . .

Tilla Weinstein, Another conformal structure on immersed surfaces of negative

curvature.

Gregers Louis Krabbe, Spectral permanence of scalar operators

Shige Toshi Kuroda, Finite-dimensional perturbation and a representaion of

scattering operator.

Marvin David Marcus and Afton Herbert Cayford, Equality in certain

inequalities

Joseph Martin, A note on uncountably many disks .

Eugene Kay McLachlan, Extremal elements of the convex cone of semi-norms . . . . 1335

John W. Moon, An extension of Landau's theorem on tournaments . .

Louis Joel Mordell, On the integer solutions of $y(y+1)=x(x$

Kenneth Roy Mount, Some remarks on Fitting's invariants .....

Miroslav Novotný, Über Abbildungen von Mengen ............

Robert Dean Ryan, Conjugate functions in Orlicz spaces.

John Vincent Ryff, On the representation of doubly stochastic operators . . . . . . . . 1379

Donald Ray Sherbert, Banach algebras of Lipschitz functions .

James McLean Sloss, Reflection of biharmonic functions across analytic boundary

conditions with examples.

L. Bruce Treybig, Concerning homogeneity in totally ordered, connected topological space....

John Wermer, The space of real parts of a function algebra...

James Juei-Chin Yeh, Orthogonal developments of functionals and related theorems

in the Wiener space of functions of two variables......... 